\title{
FUNGOS MICORRÍZICOS ARBUSCULARES EM BANANEIRAS CULTIVADAS NO VALE DO SUBMÉDIO SÃO FRANCISCO ${ }^{1}$
}

\author{
Adriana Mayumi Yano de $\mathrm{Melo}_{3}^{2}$ \\ Leonor Costa Maia ${ }^{3}$ \\ Luiz Balbino Morgado
}

Recebido em 28/5/97. Aceito em 18/10/97

\begin{abstract}
RESUMO - (Fungos micorrizicos arbusculares em bananeiras cultivadas no Vale do Submédio São Francisco). Na região do Vale do São Francisco, onde a exploração da agricultura irrigada é a principal responsável pela alta produção de frutas para exportação, a banana é uma das culturas que mais tem se expandido em área cultivada. Com o objetivo de registrar os fungos micorrizicos arbusculares associados a essa cultura, foram coletadas, sob a parte aérea da bananeira, amostras de solo da rizosfera, em quatro áreas com diferentes características de solo, sendo identificadas as espécies: Acaulospora longula Spain \& Schenck, A. morrowiae Spain \& Schenck, A. rehmii Sieverding \& Toro, A. scrobiculata Trappe, A. spinosa Walker \& Trappe, A. tuberculata Janos \& Trappe, Entrophospora infrequens (Hall) Ames \& Schneider, Gigaspora ramisporophora Spain, Sieverding \& Schenck, Glomus diaphanum Morton \& Walker, G. etunicatum Becker \& Gerd., G. intraradices Schenck \& Smith, G. macrocarpum Tul. \& Tul., G. mosseae (Nicol. \& Gerd.) Gerd. \& Trappe, G. occultum Walker e Scutellospora sp. A percentagem de colonização das raízes de bananeiras nas diferentes áreas foi em média $55 \%$.
\end{abstract}

Palavras-chave: endomicorriza, Musa spp., Glomales, semi-árido, biodiversidade, áreas irrigadas

\begin{abstract}
Arbuscular mycorrhizal fungi associated with roots of banana cultivated in the Submédio São Francisco Valley). In the region of the São Francisco Valley, where irrigated agriculture is responsable for high production of fruits for exportation, banana is one of the most expanded crops in cultivated areas. In order to know the arbuscular mycorrhizal fungi associated with this crop, samples from the rhizosphere of banana plants were collected in four areas with different soil characteristics, being identified the species: Acaulospora longula Spain \& Schenck, A. morrowiae Spain \& Schenck, A. rehmii Sieverding \& Toro, A. scrobiculata Trappe, A. spinosa Walker \& Trappe, A. tuberculata Janos \& Trappe, Entrophospora infrequens (Hall) Ames \& Schneider, Gigaspora ramisporophora Spain, Sieverding \& Schenck, Glomus diaphamum Morton \& Walker, G. etunicatum Becker \& Gerd., G. intraradices Schenck \& Smith, G. macrocarpum Tul. \& Tul., G. mosseae (Nicol. \& Gerd.) Gerd. \& Trappe, G. occultum Walker and Scutellospora sp. The rate of colonization of banana roots by arbuscular mycorrhizal fungi in the investigated areas was around $55 \%$.
\end{abstract}

Key words: endomycorrizhae, Musa spp., Glomales, semi-arid lands, biodiversity, irrigated areas

Trabalho apresentado no XIII Congresso Latino Americano de Ciência do Solo, Águas de Lindóia, SP EMBRAPA-CPATSA, C.P. 23, CEP 56300-000, Petrolina, PE, Bolsista CNPq, DCR, e-mail: adriana@cpatsa.embrapa.br

Departamento de Micologia, Centro de Ciências Biológicas, Universidade Federal de Pernambuco, CEP 50670-420, Recife, PE, Bolsa CNPq-PP

EMBRAPA-CPATSA, C.P. 23, CEP 56300-000, Petrolina, PE 


\section{Introdução}

O Vale do Submédio São Francisco destaca-se, principalmente, pela exploração da agricultura irrigada para produção de alimentos, em especial culturas frutícolas e, a cada dia, tem suas áreas de plantio aumentadas para suprir a demanda tanto para consumo interno como para exportação. A bananeira, uma das fruteiras com extensas áreas plantadas nessa região, alcança boa produtividade, em decorrência das condições climáticas e edáficas propícias.

Uma vez que a demanda por mudas de bananeira nesta região é elevada, a rápida produção destas poderá suprir as necessidades do mercado. Fungos micorrízicos arbusculares (FMA) são amplamente conhecidos por aumentar a absorção de nutrientes, principalmente fósforo, ocasionando maior incremento no crescimento das plantas (Siqueira 1994). Desta forma, podem ser utilizados para reduzir o tempo de formação das mudas e aumentar a sobrevivência e o crescimento das mesmas após o transplante, garantindo a produção de plantas mais sadias e produtivas.

Segundo Saggin Jr. \& Siqueira (1996) para a utilização de FMA benéficos é necessária a seleção de espécies ou isolados eficientes e adaptados às condições edafoclimáticas. Assim, o levantamento de fungos nativos no ecossistema em questão é o primeiro passo para a seleção de espécies eficientes em promover o crescimento da bananeira.

Poucos trabalhos relatam a associação da bananeira com FMA (Iyer et al .1988; Jaizme-Vega \& Azcón 1995; Declerck et al. 1995). Do mesmo modo, tem sido ressaltada a escassez de trabalhos em taxonomia de fungos em ecossistemas de caatinga (Trufem 1996).

Este trabalho teve como objetivos o levantamento dos FMA ocorrentes em rizosfera de bananeiras (cultivar pacovan) cultivadas sob irrigação no Vale do Submédio São Francisco, e a observação do grau de colonização natural de raízes dessas plantas por tais fungos simbiontes, para posterior estudo do efeito da inoculação de FMA sobre a produção de mudas.

\section{Material e métodos}

Áreas de estudo e coleta das amostras de solo - quatro áreas, com solos que apresentam características químicas diferentes (Tab. 1), foram selecionadas para coleta das amostras de solo, sendo elas: 1. BEBEC - Projeto de Irrigação Bebedouro - Estação Experimental do Centro de Pesquisa Agropecuária do Trópico Semi-Árido (CPATSA) da Empresa Brasileira de Pesquisa Agropecuária (EMBRAPA); 2. IPA - Projeto de Irrigação Nilo Coelho, em área pertencente à Empresa Pernambucana de Pesquisa Agropecuária, onde se encontra a área Experimental do CPATSA-EMBRAPA; 3. BEBPA - Projeto de Irrigação Bebedouro - Lote Patrícia e 4. MAC - Projeto de Irrigação Mandacaru. Com exceção desta última, localizada em Juazeiro, Bahia, em Vertissolo, as demais áreas encontram-se em Petrolina, Pernambuco, em Latossolo. As amostras de solo foram coletadas com o auxílio de um trado até a profundidade de $20 \mathrm{~cm}$ da superficie do solo, totalizando, em cada área, vinte amostras compostas de cinco subamostras. 
Tabela 1 - Características químicas de quatro solos cultivados com bananeiras, em áreas irrigadas do Submédio São Francisco.

\begin{tabular}{|c|c|c|c|c|c|c|c|c|}
\hline$\overline{\text { SOLO }}$ & $\mathrm{pH}-\mathrm{H}_{2} \mathrm{O}$ & C.E. $/ 25^{\circ} \mathrm{C}$ & $\mathrm{Ca}$ & $\mathrm{Mg}$ & $\mathrm{Na}$ & $\mathrm{K}$ & $\mathrm{Al}$ & $\mathrm{P}^{* *}$ \\
\hline (Local) & $1: 2,5$ & $(\mathrm{dS} / \mathrm{m})$ & \multicolumn{5}{|c|}{$\left(\mathrm{cmol} / \mathrm{dm}^{3}\right)$} & $\left(\mathrm{mg} / \mathrm{dm}^{3}\right)$ \\
\hline BEBEC & 5,65 & 0,92 & 1,70 & 1,40 & 0,05 & 0,57 & 0,07 & 31 \\
\hline IPA & 6,7 & 0,65 & 4,20 & 1,10 & 0,04 & 0,51 & 0,02 & 46 \\
\hline MAC & 7,40 & 0,46 & 2,45 & 1,25 & 0,02 & 0,22 & 0,00 & 53 \\
\hline BEBPA & 6,5 & $2,52^{*}$ & 7,15 & 0,90 & 0,56 & 0,39 & 0,05 & 22 \\
\hline
\end{tabular}

* Na profundidade de $0-10 \mathrm{~cm}$ a Condutividade Elétrica (C.E.) foi em torno de $4,2 \mathrm{dS}^{-1} \mathrm{~m}$.

** Extrator Mehlich - 1. (BEBEC= Projeto de Irrigação Bebedouro; IPA= Projeto de Irrigação Nilo Coelho; $\mathrm{MAC}=$ Projeto de Irrigação Mandacaru e BEBPA= Lote Patricia).

Multiplicação dos FMA - as vinte amostras de cada área foram homogeneizadas, adicionando-se areia e vermiculita esterilizadas na proporção 2:1:1 (solo: areia: vermiculita) e colocadas em vasos com capacidade para 10 litros, para multiplicação dos FMA presentes nos solos, sendo utilizada como planta hospedeira o sorgo (Sorghum vulgare L.) de forma a se obter maior quantidade de esporos viáveis (Melville 1993). Os vasos foram mantidos por três meses em casa de vegetação, a temperatura média de $25^{\circ} \mathrm{C}$, com irrigação em dias alternados.

Identificação dos FMA e contagem do número de esporos - para identificação e contagem da população de esporos de FMA, fez-se a extração dos mesmos pela técnica de peneiramento por via úmida (Gerdemann \& Nicolson 1963) e centrifugações em água destilada e, posteriormente, em sacarose $40 \%$. Os esporos retidos nas peneiras foram transferidos para placas de Petri e contados com auxílio de estereomicroscópio. Esporos foram montados entre lâmina e lamínula com PVLG (álcool-polivinílicolactoglicerol) (Schenck \& Pérez 1990). A bibliografia utilizada para identificação das espécies foi, basicamente, a de Schenck \& Perez (1990).

Colonização micorrízica - para observação da colonização das raízes de bananeira por FMA, utilizou-se o método de clarificação e coloração proposto por Phillips \& Hayman (1970). A avaliação da percentagem de colonização foi feita pelo método da intersecção de quadrantes (Giovannetti \& Mosse 1980).

\section{Resultados e discussão}

Levantamento taxonômico - foi observada a presença de quinze espécies de FMA associadas a raízes de bananeiras, nas áreas estudadas (Tab. 2). Destas, somente Glomus macrocarpum havia sido anteriormente mencionada em associação com raízes de bananeira por lyer et al. (1988), que referiram, além desta espécie, a presença de G. fuegianum, Gigaspora decipens e G. heterogama (= Scutellospora heterogama).

Ressalta-se a presença em maior quantidade de esporos das espécies Acaulospora scrobiculata e Glomus mosseae em quase todas as amostras de solo coletadas. Essas espécies foram referidas por Maia \& Trufem (1990) e Farias (1994) em solos do sertão nordestino. Vale salientar que $A$. scrobiculata também foi observada em cultura de banana (Musa sp.), em solos de Minas Gerais, por Siqueira et al. (1989). A predominância e ocorrência generalizada de $A$. scrobiculata nas diferentes áreas de coleta e a sua ampla distribuição em vários ecossistemas brasileiros (Bononi \& Trufem 1983; Trufem \& Bononi 1985; Schenck et al. 1986; Schenck \& Siqueira 1987) foram 
também referidas por Lopes et al. (1983), Fernandes \& Siqueira (1989) e Siqueira (1994), que encontraram esta espécie em cafezais e em outros ecossistemas agrícolas de Minas Gerais. Segundo Siqueira et al. (1989), A. scrobiculata parece ser favorecida por baixos teores de alumínio e altos teores de fósforo no solo, sugerindo adaptação a condições de solo cultivado.

Tabela 2 - Táxons de FMA verificados em solos plantados com bananeiras, em áreas irrigadas do Submédio São Francisco.

\begin{tabular}{|c|c|c|c|c|}
\hline \multirow[t]{2}{*}{ Espécies } & \multicolumn{4}{|c|}{ Locais de coleta } \\
\hline & BEBEC & IPA & MAC & BEBPA \\
\hline Acaulospora longula Spain \& Șchenck & - & - & - & + \\
\hline A. morrowiae Spain \& Schenck & + & - & - & - \\
\hline A. rehmii Sieverding \& Toro & - & + & - & - \\
\hline A. scrobiculata Trappe & + & + & + & + \\
\hline A. spinosa Walker \& Trappe & + & - & - & - \\
\hline A, tuberculata Janos \& Trappe & - & + & - & + \\
\hline Entrophospora infrequens (Hall) Ames \& Schneider & - & - & - & + \\
\hline Gigaspora ramisporophora Spain, Sieverding \& Schenck & - & - & + & - \\
\hline Glomus diaphanum Morton \& Walker & - & - & - & + \\
\hline G. etunicatum Becker \& Gerd. & + & + & - & - \\
\hline G. intraradices Schenck \& Smith & - & - & - & + \\
\hline G. macrocarpum Tul. \& Tul. & - & + & - & + \\
\hline G. mosseae (Nicol. \& Gerd.) Gerd. \& Trappe & - & + & + & + \\
\hline G. occultum Walker & - & - & + & + \\
\hline Scutellospora sp. & - & - & + & - \\
\hline
\end{tabular}

$\overline{(\mathrm{BEBEC}}=$ Projeto de Irrigação Bebedouro; $\mathrm{IPA}=$ Projeto de Irrigação Nilo Coelho; $\mathrm{MAC}=$ Projeto de Irrigação Mandacaru e $\mathrm{BEBPA}=$ Lote Patricia).

A ocorrência de Glomus mosseae nos solos de MAC, IPA e BEBPA, onde o pH apresentou valores médios entre 6,5 a 7,4, confirma a observação de Schenck \& Siqueira (1987) e de Schenck \& Pérez (1990) em relação à preferência desta espécie por solos neutros a levemente alcalinos. Vale salientar que essa espécie tem ampla distribuição, havendo sido referida em grande número de países, tais como Nigéria, India, Finlândia, Venezuela, Filipinas, Indonésia, Estados Unidos, União Soviética e Dinamarca (Dodd et al. 1996), entre outros, o que indica elevada capacidade para sobrevivência em diferentes ecossistemas. Dodd \& Krikun (1984) mencionaram que isolados de G. mosseae, procedentes de solos arenosos e calcáreos do deserto de Negev (Israel), foram capazes de germinar em ágar-água suplementado ou não com nutrientes e com pH variando desde 0,5 até 8,0 . Por outro lado, Daniels \& Graham (1976) verificaram que excesso de nutrientes pode ser prejudicial à germinação da espécie.

Gigaspora ramisporophora destaca-se por ter sido coletada no solo do MAC, ao norte da Bahia, região de clima semi-árido. Essa espécie foi descrita pela primeira vez em solos do sul deste Estado, área caracterizada por clima litorâneo, onde se cultiva predominantemente o cacaueiro (Spain et al.1989).

Schenck \& Siqueira (1987) constataram que espécies do gênero Gigaspora ocorriam em solos com pH menor ou igual a 5,3, enquanto a maioria das espécies dos gêneros Glomus e Acaulospora ocorriam em pH maior ou igual a 6,1. Entretanto, esta 
relação não foi confirmada no presente trabalho, salientando-se que a única espécie de Gigaspora observada ocorreu em solo alcalino (Tab. 1 e 2).

No solo da BEBPA, embora apresentando condutividade elétrica elevada, com tendência a salinização (Tab. 1), observou-se maior diversidade de espécies. Nas demais áreas a diversidade foi menor: IPA, MAC e BEBEC, com seis, cinco e quatro espécies, respectivamente.

Acaulospora spinosa, A. tuberculata, Glomus etunicatum e $G$. intraradices não haviam sido registradas anteriormente em solos do Estado de Pernambuco. Entre as espécies observadas, A. spinosa, A. tuberculata, Entrophospora infrequens, Glomus diaphanum, G. etunicatum, G. intraradices e G. macrocarpum foram encontradas em outros ecossistemas, no Sudeste do país (Trufem \& Bononi 1985).

As espécies Acaulospora longula e A. morrowiae, anteriormente citadas por Siqueira et al. (1989) em plantas nativas no Estado de Minas Gerais e por Weber \& Oliveira (1994) em solos plantados com citros nos Estados da Bahia e Sergipe, também foram encontradas neste levantamento.

Percentagem de colonização e número de esporos - a percentagem de colonização de raizes, por FMA, nos bananais estudados no Vale do Submédio São Francisco foi relativamente alta, em torno de 55\% (Tab. 3), embora os solos de todas as áreas de coleta tenham apresentado alto teor de fósforo (Tab.1) o que normalmente diminui a colonização micorrízica (Abbott \& Robson 1991; Brundrett 1991). Desta forma, a percentagem de colonização parece não ter sido inibida pelo fósforo disponível, visto que esta apresentou níveis semelhantes aos obtidos por Iyer et al. (1988), em condições naturais e variaram pouco em relação às diferentes áreas de coleta.

Tabela 3 - Número de esporos na rizosfera e percentagem de colonização de raizes por FMAs em solos irrigados do Submédio São Francisco, cultivados com bananeiras.

\begin{tabular}{lcccc}
\hline Parâmetros & \multicolumn{4}{c}{ Locais de Coleta } \\
\cline { 2 - 5 } & BEBEC & IPA & MAC & BEBPA \\
\hline Densidade de esporos* & 44 & 134 & 271 & 228 \\
Colonização (\%) & 53,33 & 58,66 & 57,33 & 54,66 \\
\hline
\end{tabular}

* Número de esporos em $100 \mathrm{~g}$ de solo. (BEBEC= Projeto de Irrigação Bebedouro; IPA= Projeto de Irrigação Nilo Coelho; $\mathrm{MAC}=$ Projeto de Irrigação Mandacaru e BEBPA= Lote Patrícia).

Siqueira (1994) afirma que os níveis de fósforo no solo interferem na colonização e na esporulação dos FMA, sendo que os efeitos deste nutriente na colonização são diferentes entre as espécies, pois atuam via nutrição da planta; por isso, a quantidade de nutrientes suficiente para inibir a colonização depende da capacidade de absorção e translocação da espécie vegetal.

Segundo Declerck et al. (1995), algumas variedades de banana apresentam alto grau de dependência micorrízica, o que explica a elevada colonização alcançada por FMA nesta fruteira. Desta maneira, os níveis de colonização micorrizica superiores a $50 \%$, obtidos no presente estudo, podem sugerir grande dependência do cultivar pacovan à micorrização.

Em relação à quantidade de esporos de FMA, os solos coletados apresentaram grande variação. O solo da área BEBEC apresentou a menor densidade, com cerca de 44 esporos/100g de solo, enquanto o solo da área MAC teve o maior número, com 
271 esporos/100g de solo (Tab. 3). Embora o número de esporos no solo da área BEBEC seja menor que nos demais, a percentagem de colonização das raizes de bananeira por FMA não variou entre os locais, confirmando o trabalho de Maia \& Trufem (1990) que também não observaram relação entre o número de esporos de FMA no solo e a colonização micorrízica.

Foi observado que o solo da área BEBEC era o de $\mathrm{pH}$ mais baixo $(5,65)$, em comparação com os demais solos estudados e que nessa área tanto o número de esporos quanto a diversidade de espécies e a colonização micorrizica foram também os que apresentaram valores menores. Possivelmente o $\mathrm{pH}$ exerce influência decisiva na formação da associação micorrízica nessas áreas.

\section{Agradecimentos}

Ao Sidney L. Sturmer (INVAM, West Virginia University), pela identificação de algumas espécies de FMA coletadas; a Orivaldo J. Saggin Júnior (EMBRAPA Centro de Pesquisa Agropecuária do Trópico Semi-Árido-CPATSA), pelas críticas e sugestões; ao CNPq, pelo suporte financeiro (Processo n ${ }^{\circ}$ 300939/95-7) e à EMBRAPACPATSA, pelo auxílio (08.0.95.101-04) e permissão do uso de suas instalações.

\section{Referências bibliográficas}

Abbott, L.K. \& Robson, A.D. 1991. Factors influencing the occurrence of vesicular-arbuscular mycorrhizas. Agriculture, Ecosystems and Environment 35: 121-150.

Brundrett, M. 1991. Mycorrhizas in natural ecosystems. Advances in Ecological Research 21: 171-313.

Bononi, V.L.R. \& Trufem, S.F.B. 1983. Endomicorrizas vesículo-arbusculares do cerrado da Reserva Biológica de Moji-Guaçu, São Paulo, Brasil. Rickia 10: 55-84.

Daniels, B.A. \& Graham, S.O. 1976. Effects of nutrition and soil extracts on germination of Glomus mosseae spores. Mycologia 68: 108-116.

Declerck, S.; Plenchette, C. \& Strullu, D.G. 1995. Mycorrhizal dependency of banana (Musa acuminata, AAA group) cultivar. Plant and Soil 176: 183-187.

Dodd, J. \& Krikun, J. 1984. Observations on Endogonaceous spores in the Negev desert. Transactions of the British Mycological Society 82(3): 536-540.

Dodd, J.C.; Rosendahl, S.; Giovanetti, M.; Broome, A.; Lanfranco, L. \& Walker, C. 1996. Inter-and intraspecific variation within the morphologically-similar arbuscular mycorrhizal fungi Glomus mosseae and Glomus coronatum. New Phytologist 133(1): 113-122.

Farias, M.M.C. 1994. Efeito de fungos micorrízicos arbusculares (FMA) e da adição de fósforo sobre o desenvolvimento do milho (Zea mays L.), cultivado em solos com diferentes sistemas de manejo (Serra Talhada-PE). Recife. Universidade Federal de Pernambuco.Dissertação de Mestrado.

Fernandes, A.B. \& Siqueira, J.O. 1989. Micorrizas vesicular-arbusculares em cafeeiros da região sul do Estado de Minas Gerais. Pesquisa Agropecuária Brasileira 24(12): 1489-1498.

Gerdemann, J.W. \& Nicolson, T.H. 1963. Spores of mycorrhizal Endogone species extracted from soil by wet sieving and decanting. Transactions of the British Mycological Society 46(2): 235-244.

Giovannetti, M. \& Mosse, B. 1980. An evaluation of techniques for measuring vesicular arbuscular mycorrhizal infection in roots. New Phytologist 84(3): 489-500.

Iyer, R.; Moosa, H. \& Kalpana Sastry, R. 1988. Vesicular-arbuscular mycorrhizal association in banana. Current Science 57(3): 153-155.

Jaizme-Vega, M.C. \& Azcón, R. 1995. Responses of some tropical and subtropical cultures to endomycorrhizal fungi. Mycorrhiza 5: 213-217.

Lopes, E.S.; Oliveira, E.; Dias, R. \& Schenck, N.C. 1983. Occurrence and distribution of vesicular-arbuscular mycorrhizal fungi in coffee (Coffea arabica L.) plantations in Central São Paulo State, Brazil. Turrialba 33(4): 417-422. 
Maia, L.C. \& Trufem, S.F.B. 1990. Fungos micorrizicos vesículo-arbusculares em solos cultivados no Estado de Pernambuco. Revista Brasileira de Botânica 13(2): 89-95.

Phillips, J.M. \& Hayman, D.S. 1970. Improved procedures for clearing roots and staining parasite and vesiculararbuscular mycorrhizal fungi for rapid assessment of infection. Transactions of the British Mycological Society 55(1): 158-161.

Saggin Jr., O.J. \& Siqueira, J.O. 1996. Micorrizas arbusculares em cafeeiro. Pp. 203-254. In: J.O. Siqueira (Ed.), Avanços em fundamentos e aplicação de micorrizas. Lavras. Universidade Federal de LavrasDCS e DCF.

Schenck, N.C. \& Perez, Y. 1990. Manual for the identification of VA mycorrhizal fungi. Gainesville, Florida: Synergistic Publ.

Schenck, N.C \& Siqueira, J.O, 1987. Ecology of VA mycorrhizal fungi in temperate agroecosystems. Pp. 2-4. In: D.M. Sylvia; L.L. Hung \& J.H. Graham (Eds.), Mycorrhizae in the Next Decade. $7^{\text {th }}$ North American Conference on Mycorrhizae. Gainesville, Florida.

Schenck, N.C; Siqueira, J.O. \& Oliveira, E. 1986. Incidence of VA mycorrhizal fungal species in some native and cultivated cerrado soils of Minas Gerais State. Fitopatologia Brasileira 11(2): 350-351.

Siqueira, J.O. 1994. Micorrizas. Pp. 151-194. In: R.S. Araújo \& M. Hungria (Eds.), Microrganismos de importância agrícola. Brasília: EMBRAPA-CNPAF, EMBRAPA-CNPSo, EMBRAPA-SPI.

Siqueira, J.O.; Colozzi-Filho, A. \& Oliveira, E. 1989. Ocorrência de micorrizas vesicular-arbusculares em agro e ecossistemas do Estado de Minas Gerais. Pesquisa Agropecuária Brasileira 24(12): 1499-1506.

Spain, J.L.; Sieverding, E. \& Schenck, N.C. 1989. Gigaspora ramisporophora: a new species with novel sporophores from Brazil. Mycotaxon 34: 667-677.

Trufem, S.F.B. 1996. Methods for the assessment of diversity in mycorrhizae. Pp. 49-63. In: C.E. Bicudo \& N.A. Menezes (Eds.), Biodiversity in Brazil: a first approach. São Paulo: CNPq.

Trufem, S.F.B. \& Bononi, V.L. 1985. Micorrizas vesiculo-arbusculares de culturas introduzidas em áreas de cerrado. Rickia 12: 165-187.

Weber, O.B. \& Oliveira, E. 1994. Ocorrência de fungos micorrizicos vesículo-arbusculares em citros nos Estados da Bahia e Sergipe. In: Resumo do III Simpósio Brasileiro sobre Microbiologia do Solo. Resumo...Londrina: SBM. p. 113-A. 